\title{
Calcium polystyrene sulfonate bezoar in the ileum: diagnosis and treatment with double-balloon endoscopy
}

An 86-year-old man, admitted for transarterial chemoembolization of a hepatoma, developed fever and acute renal failure following the procedure. He was given antibiotic therapy and oral calcium polystyrene sulfonate ( $30 \mathrm{~g}$ daily) for hyperkalemia. After 1 week, the patient developed bilious vomiting with abdominal pain. An abdominal X-ray disclosed diffuse dilatation of the small bowel ( $\bullet$ Fig. 1) and computed tomography was suspicious for a bezoar in the ileum along with intestinal obstruction ( Fig.2). A surgeon was consulted but surgical therapy was declined due to the high surgical risk. The patient received conservative medical therapy but his intestinal obstruction failed to resolve. A decision was made to carry out retrograde enteroscopy to emove the bezoar. When the ileum was entered, we found a large, tubular-shaped, firm yellowish bezoar occupying the intestinal lumen $(\bullet$ Fig. 3 and $\bullet$ Video 1$)$. There were also some ileal ulcers. Irrigation with water and fragmentation using a snare helped mobilize the bezoar. Multiple small brownish granules were seen after the bezoar was fragmented ( $\bullet$ Fig.4) and the aspirated fluid contained resin granules. Given the endoscopic findings and the drug history of the patient, he was diagnosed as having a calcium polystyrene sulfonate resin-associated bezoar. Despite the efforts to remove the bezoar, the patient died of multiple organ failure 1 month later.

Calcium polystyrene sulfonate is an exchange resin used to treat hyperkalemia. A few cases of resin-related bezoars with intestinal obstruction have been reported [1-3]. Such bezoars most often form in critically ill infants [1-3] or in debilitated elderly patients with decreased bowel mobility and prolonged usage of the agent. The treatment of polystyrene sulfonate-related intestinal obstruction is surgery; only one case of non-surgical management has been reported [3]. The present case report documents the endoscopic findings related to resin-associated bezoar. In addition, we have found enteroscopy may be a useful tool in the treatment of intestinal obstruction resulting from the presence of such a bezoar.

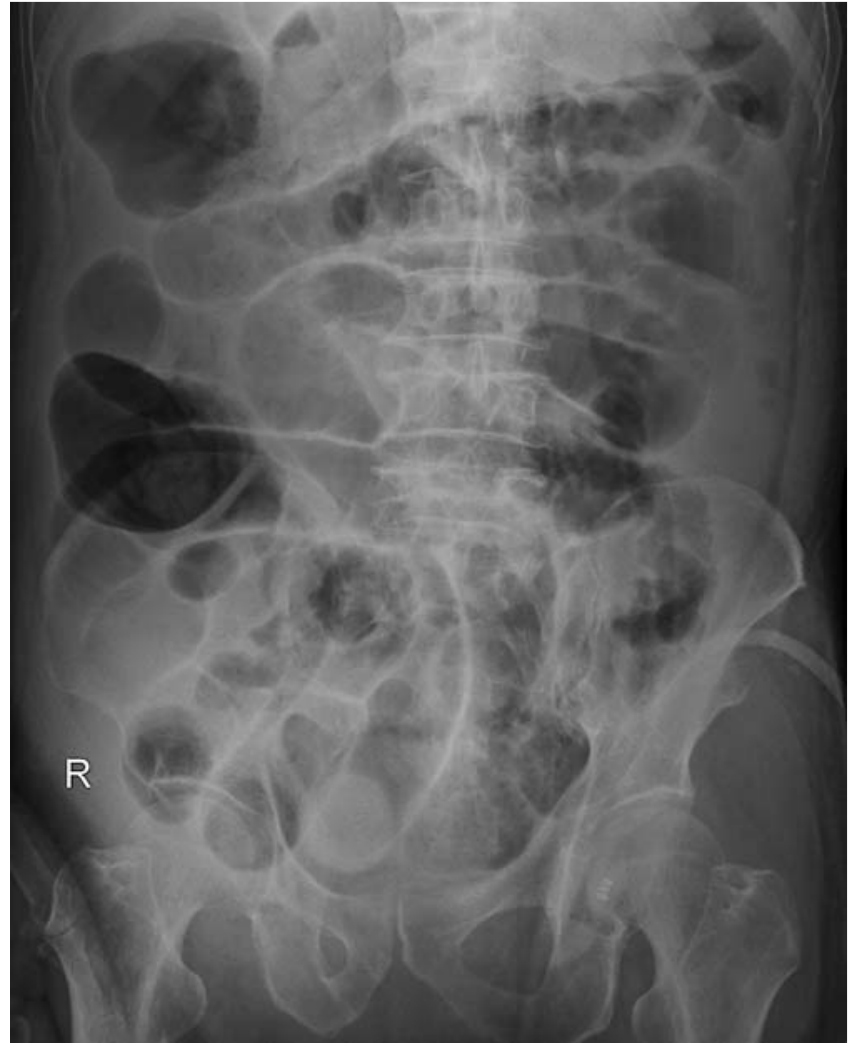

Fig. 1 Abdominal $X$-ray showing diffuse small-bowel dilatation in an 86-year-old man with a drug-induced bezoar.

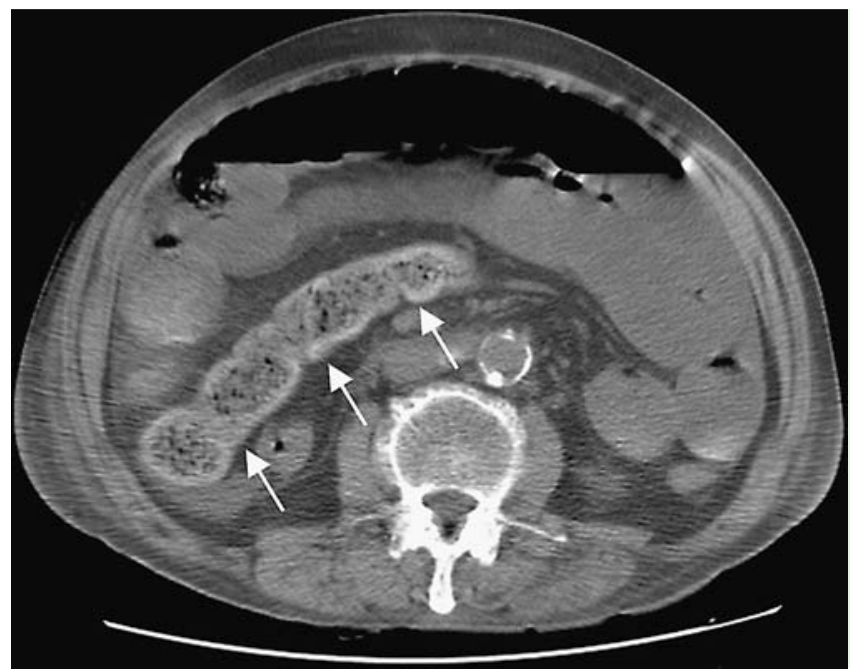

Fig.2 Abdominal computed tomography (CT) showing radiopaque material (arrows) in the ileum and which caused the intestinal obstruction.

Video 1

A calcium polystyrene sulfonate resin-associated bezoar in the small intestine removed by endoscopic fragmentation.
Endoscopy_UCTN_Code_TTT_1AP_2AD

Competing interests: None 


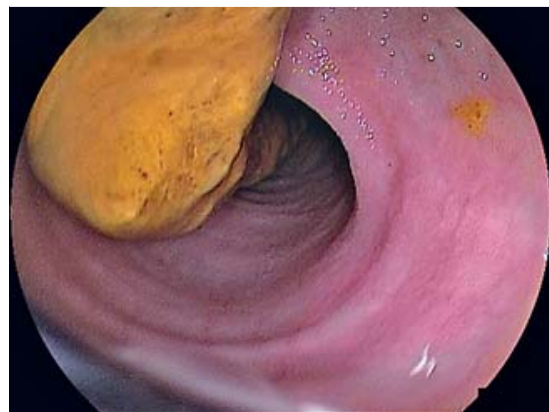

Fig. 3 Endoscopic view showed a large, tubular and firm, yellowish structure occupying the ileal lumen.

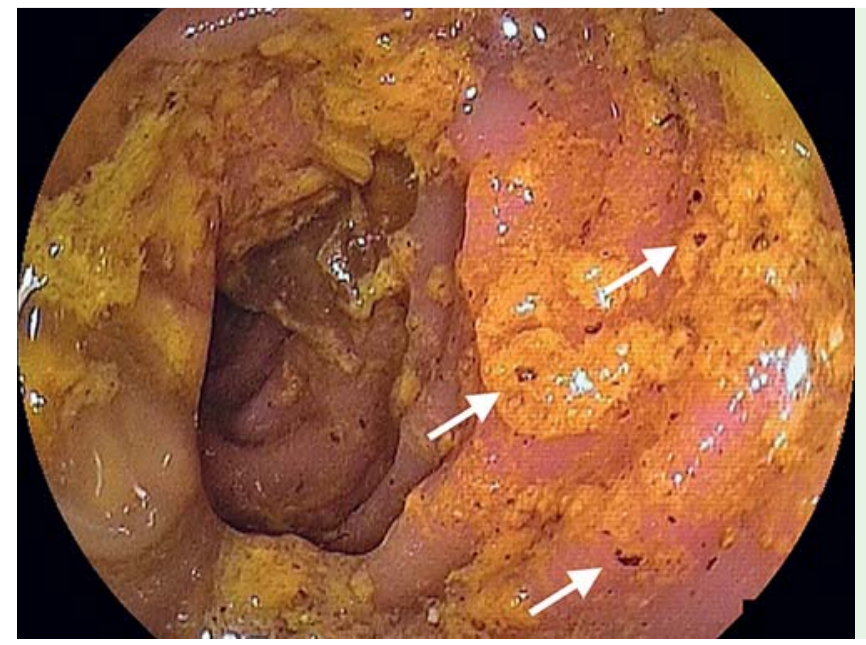

Fig. 4 Post-fragmentation endoscopic view of the bezoar. Multiple small granules (arrows) were observed.
Tien P. Lai ${ }^{1}$, Chia W. Yang ${ }^{2}$, Fu Y. Siaop ${ }^{1}$, Hsu H. Yen ${ }^{2}$

${ }^{1}$ Department of Emergency Medicine, Changhua Christian Hospital, Changhua, Taiwan, Republic of China

2 Department of Gastroenterology, Changhua Christian Hospital, Changhua, Taiwan, Republic of China

\section{References}

1 Metlay LA, Klionsky BL. An unusual gastric bezoar in a newborn: Polystyrene resin and candida albicans. J Pediatr 1983; 102: 121 123

2 Garcia-Pardo G, Martinez-Vea A, Auguet T et al. Intestinal obstruction complicating calcium polystyrene sulphonate therapy. Nephrol Dial Transplant 1996; 11: 751

3 Koneru P, Kaufman RA, Talati AJ et al. Successful treatment of sodium PolyStyrene sulfonate bezoars with serial water-soluble contrast enemas. J Perinatol 2003; 23: $431-433$

\section{Bibliography}

DOI http://dx.doi.org/

10.1055/s-0033-1344835

Endoscopy 2013; 45: E378-E379

(c) Georg Thieme Verlag KG

Stuttgart · New York

ISSN 0013-726X

\section{Corresponding author}

\section{Hsu H. Yen}

Endoscopy Center

Department of Gastroenterology

Changhua Christian Hospital

Changhua City

135 Nanhsiao Street Changhua

500 Taiwan

China

91646@cch.org.tw 